\title{
Dislocation-Limited Performance of Advanced Solar Cells through TCAD Modeling
}

\author{
David Berney Needleman ${ }^{1}$, Hannes Wagner ${ }^{1}$, Pietro P. Altermatt ${ }^{2}$, Zhen Xiong ${ }^{2}$, Pierre J. \\ Verlinden $^{2}$, and Tonio Buonassisi ${ }^{1}$ \\ ${ }^{1}$ Massachusetts Institute of Technology, Cambridge, MA 02139, USA \\ 2 Trina Solar, State Key Laboratory for Photovoltaic Science and Technology (SKL), No 2 \\ Trina Road, Trina PV Industrial Park, Xinbei District, Changzhou, Jiangsu Province, China
}

213031

\begin{abstract}
In recent years, low-cost silicon for photovoltaic applications has trended toward smaller grain sizes with lower dislocation densities. These changes, coupled with advanced device architectures like heterojunction and passivated emitter and rear cells, have enabled higher efficiencies, including new world records. To establish material requirements for further improvement, we model dislocations with different recombination strengths in Sentaurus Device using mid-gap defects spatially localized at the nanoscale in three dimensions. This approach accounts for the charging of dislocations and differentiates between dislocations and grain boundaries or metal impurities. Simulations that model dislocations as macroscopic variations in effective bulk lifetime or diode parameters do not address these issues. We validate our model by simulating the world record multicrystalline silicon solar cell. We find that three parameters influence cell efficiency: the area fraction of the cell containing dislocations, the dislocation density within defect clusters, and the concentration of recombination centers at these dislocations. We provide targets for these parameters and show how our results can be used to determine the potential gains from reducing dislocations in experimental devices and materials. We further predict that device architectures that lead to higher-injection, such as silicon heterojunction cells, are more robust to the presence of dislocations.
\end{abstract}

\section{INTRODUCTION}

Dislocations limit the performance of solar cells with silicon absorbers produced using a variety of established and novel techniques [1-6], including cast multicrystalline silicon (mc-Si) [7-9], seeded cast quasi-single-crystalline silicon (QSC-Si) [10,11], and kerfless silicon wafers grown epitaxially from the gas phase (epi-Si) [12]. Improvements in grainengineered, so-called "high-performance" mc-Si over the last several years have largely been the result of lower densities of recombination-active dislocations and high-energy grain boundaries [7]. Despite these gains, the performance of these materials lags behind that of traditional monocrystalline silicon produced by the Czochralski method (CZ-Si).

Technology computer aided design (TCAD) device simulations can provide insight into the relative performance of these materials, establish materials targets for high efficiency, and identify device architectures that are more tolerant to the presence of dislocations. Device simulations have typically modeled dislocations as macroscopic variations in effective bulk lifetime $[13,14]$. Bulk lifetime models do not differentiate between dislocations and other defects, such as grain boundaries or metal impurities, and neglect the charging of these defects through the occupation of spatially localized shallow and deep energy levels within the band gap.

Recently, we introduced a model with a single deep energy level governed by Shockley- 
Read-Hall statistics with modified capture cross sections for minority and majority carriers [15]. We showed that this model accurately simulates the injection-dependent recombination rate and local band bending around dislocations [15], as measured by electron-beam-induced current [16] and predicted by simulations of simple test structures (rather than full devices) that included the full density of states associated with decorated dislocations in silicon [17]. Using the method of Stokkan et al. to treat grain boundaries as arrays of dislocations [18], we used Synopsys Sentaurus Device TCAD software [19] to analyze the effect of grain boundaries on (i) industry-standard cells having a full-area rear contact with an aluminum-alloyed back-surface field (Al-BSF), (ii) on passivated emitter and rear totally diffused (PERT) cells, and (iii) on a front heterojunction cell with a gallium phosphide (GaP) emitter [20].

In the present work, we simulate the effect of homogeneously distributed dislocations and dislocation clusters on the same three devices, which are based on the models in [21]. To isolate differences in the impact of dislocations on the three devices, the PERT cell has the same front emitter diffusion as the Al-BSF cell and the same rear diffusion and contact scheme as the heterojunction cell. We simulate a GaP emitter rather than the more typical amorphous silicon so that we can optimize the front interface and emitter with regard to band alignment, optical losses, and recombination.

\section{DISLOCATION MODEL}

\subsection{Simulated domain and implementation of dislocations}

To simulate dislocations, we introduce a rectangular prism $4 \mathrm{~nm}$ square in the center of the simulated domain. $4 \mathrm{~nm}$ is chosen as typical of the size of the strain field around a dislocation core in silicon. This prism passes through the entire silicon layer perpendicular to the plane of the wafer (Fig. 1a), but not through the passivating dielectrics or the GaP emitter in the heterojunction device. We then specify an areal concentration of mid-gap recombination centers at the interface of the prism and the rest of the silicon wafer (Fig. 1b). To match the traditional description of dislocations as line defects, we report this concentration as a line density $\left(N_{\mathrm{DL}}\right)$ in units of $\mathrm{cm}^{-1}$, obtained by multiplying the areal density of recombination centers by the perimeter of the prism representing the dislocation. $N_{\text {DL }}$ determines the charging and recombination rate of the dislocation. Since there is one dislocation in the simulated domain, the dislocation density is simply $1 / \mathrm{x}^{2}$, where $\mathrm{x}$ is the lateral dimension of the (square) simulated domain (Fig. 1a).

(a) simulated domain

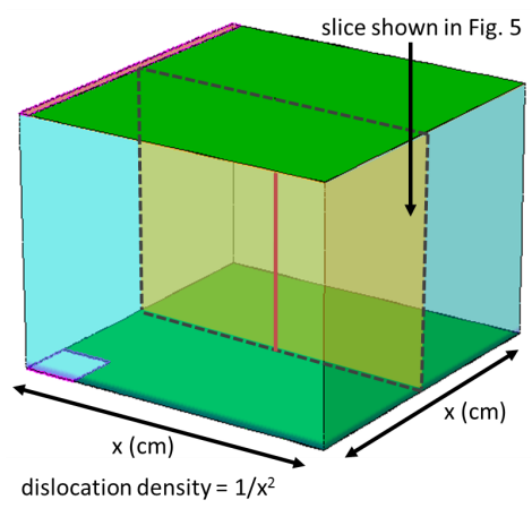

(b) enlargement of dislocation

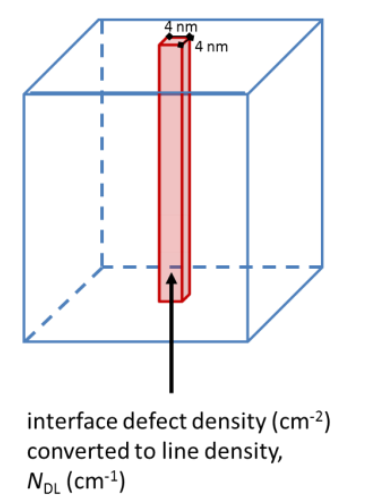

FIG. 1. Schematic of dislocation simulation. (a) The simulated domain (always square), whose lateral dimensions determine the dislocation density. (b) Enlarged schematic of the dislocation itself, modeled as a rectangular prism within the $\mathrm{Si}$ wafer. The interface between this region and the Si wafer is populated with mid-gap recombination centers. The density of these defects is multiplied by the perimeter of the prism to give a line density of recombination centers, $N_{\mathrm{DL}}$, with units $\mathrm{of}_{\mathrm{cm}}^{-1}$. 
We compare our simulated results for Al-BSF cells with experimental data from literature [22]. Fig. 2 shows our simulated efficiencies as a function of dislocation density ( $x$-axis) $N_{\text {DL }}$ (data series). We see reasonable agreement between our simulations and the literature data. Literature data falling across a range of $N_{\mathrm{DL}}$ is not surprising since the data include several Si growth techniques and only average dislocation density. Data slightly below our simulated curves is also reasonable, as we assume a $1 \mathrm{~ms}$ minority carrier lifetime away from the dislocation, which may be higher than in the experimental devices. We assume this relatively high lifetime in order to determine limiting efficiencies solely due to the presence of dislocations. We also note that lifetimes this high have been reported in several materials that have significantly higher dislocation density than $\mathrm{CZ}-\mathrm{Si}$, including epi-Si [23,24], QSC-Si [25], $n$-type mc-Si [8], and silicon grown by the so-called "noncontact-crucible method" [26,27]. While $p$-type mc-Si has not yet achieved wafer-averaged millisecond lifetimes, lifetime has increased dramatically in recent years and is now approaching $1 \mathrm{~ms}$ [28]. Furthermore, average lifetimes across an entire mc-Si wafer of greater than the $500 \mu$ s that have been reported likely indicate local lifetime greater than 1 $\mathrm{ms}$ in dislocation-free grains.

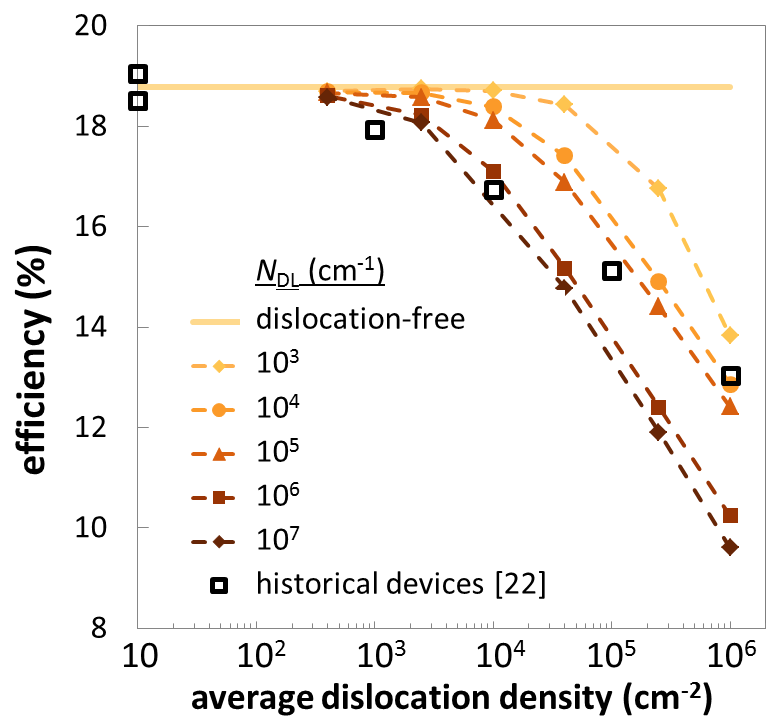

FIG. 2. Efficiency of full-area Al-BSF solar cells plotted vs. average dislocation density. Simulated efficiencies for a range of line densities of mid-gap recombination centers at the dislocation ( $N_{\mathrm{DL}}$, data series) are compared with experimental results from literature [22]. There is good agreement between simulations and literature data.

\subsection{Modelling dislocation clusters}

For the simulations shown in Fig. 2, we assumed that dislocations were homogeneously distributed throughout the cell. However, in real materials, dislocations are typically clustered. We therefore construct current-voltage $(J-V)$ curves for a solar cell containing dislocation clusters with a given dislocation density within the clusters $\left(D D_{\text {cluster }}\right)$ in units of $\mathrm{cm}^{-2}$, and a given $N_{\mathrm{DL}}\left(\mathrm{cm}^{-1}\right)$ at each individual dislocation. We construct these $J-V$ curves by taking a weighted average of the external current as a function of external voltage for the clusters and the dislocation-free regions of the cell, using the fractional area covered by each region as weights. Mathematically, this can be expressed using the formula:

$$
J_{\text {cell }}(V)=A_{\mathrm{DL}} \times J_{\mathrm{DL}}(V)+\left(1-A_{\mathrm{DL}}\right) \times J_{\text {clean }}(V),
$$


where $V$ is voltage, $J_{\text {cell }}(V)$ is the current density for the whole device as a function of voltage, $J_{\mathrm{DL}}(V)$ is the current density as a function of voltage within the dislocation cluster, $J_{\text {clean }}(V)$ is the current as a function of voltage for the dislocation-free region of the cell, and $A_{\mathrm{DL}}$ is the fraction of the total cell area containing dislocation clusters. This analysis relies on the assumption that the dislocation clusters are macroscopic, such that both the area of each cluster and the area of the dislocation-free region around the cluster are much larger than the area of interaction between them.

We test this assumption by comparing our results to a full parallel-diode model using Griddler 2.0 [29]. We fit $J-V$ curves for simulations of PERT cells without dislocations and with dislocation density of $10^{6} \mathrm{~cm}^{-2}$ to the two-diode equation. We then input these simulations into Griddler with area fractions of $10 \%$ and 50\%. In both cases, the size of individual dislocation clusters is $15.6 \mathrm{~mm} \times 15.6 \mathrm{~mm}$. The spacing of the dislocation clusters is shown in Fig. 3a,b. Fig. 3b,c and Table I show the excellent agreement between these results and the results from equation (1). Recent results from Haug et al. confirm that this result is quite general [30].

(a) $10 \%$ area fraction

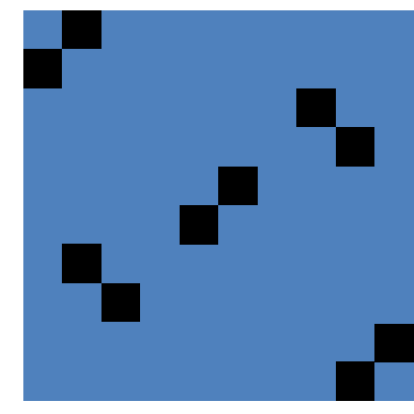

(c)

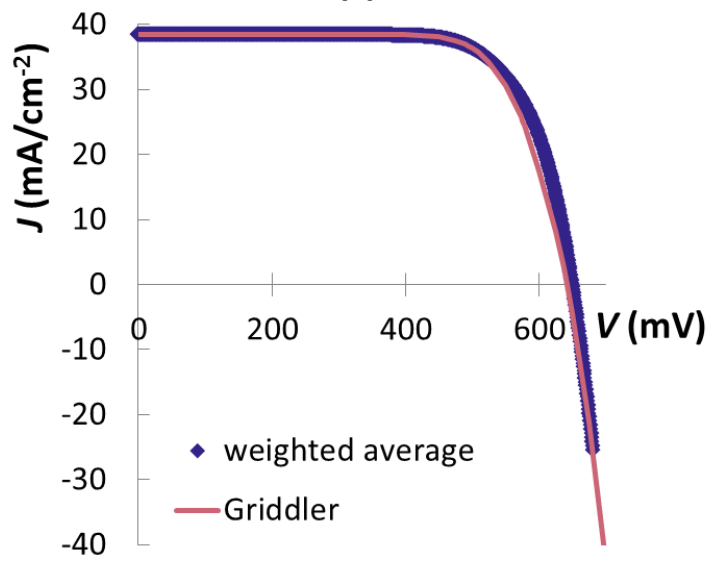

(b) $\mathbf{5 0 \%}$ area fraction

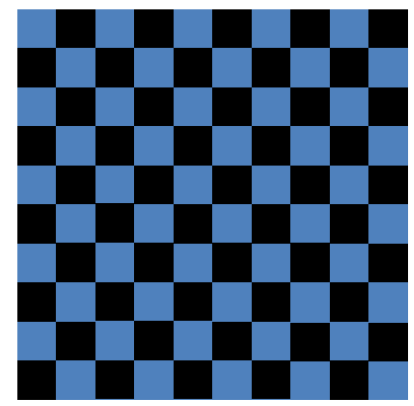

(d)

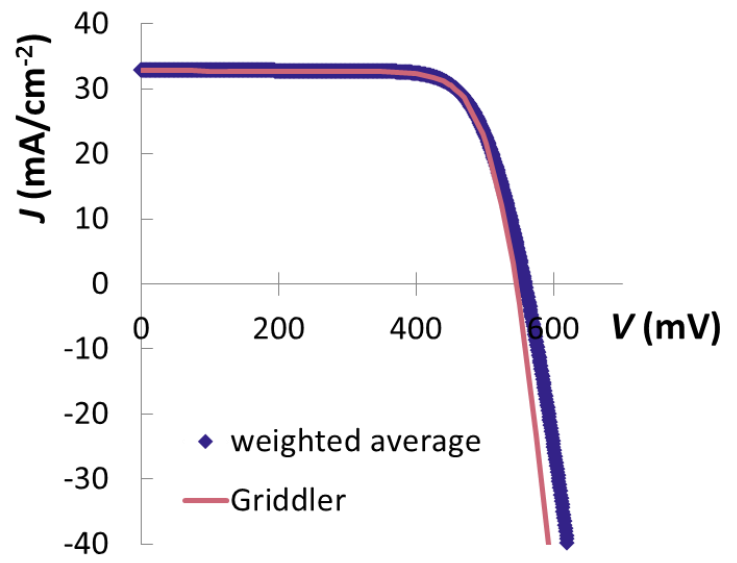

FIG. 3. (a,b) Dislocation cluster distribution for $10 \%$ and $50 \%$ coverage of dislocation clusters. (c,d) $J-V$ curves for PERT cell with $10 \%$ and $50 \%$ coverage of dislocation clusters with dislocation density of $10^{6} \mathrm{~cm}^{-2}$ simulated by taking the weighted average of the $J$ - $V$ curves of the dislocated and dislocation-free regions as described by equation (1) (purple diamonds) and a full parallel diode model using Griddler 2.0 (pink lines). Good agreement between weighted average and parallel diode simulations is reached. 
TABLE I: Comparison of weighted average and parallel diode simulations of dislocation clusters.

\begin{tabular}{|c|c|c|c|c|c|c|}
\hline & \multicolumn{2}{|c|}{ Weighted average } & \multicolumn{2}{c|}{ Griddler } & \multicolumn{2}{c|}{ Relative difference } \\
\cline { 2 - 7 } & $\begin{array}{c}10 \% \text { area } \\
\text { fraction }\end{array}$ & $\begin{array}{c}50 \% \text { area } \\
\text { fraction }\end{array}$ & $\begin{array}{c}10 \% \text { area } \\
\text { fraction }\end{array}$ & $\begin{array}{c}50 \% \text { area } \\
\text { fraction }\end{array}$ & $\begin{array}{c}10 \% \text { area } \\
\text { fraction }\end{array}$ & $\begin{array}{c}50 \% \text { area } \\
\text { fraction }\end{array}$ \\
\hline Efficiency $(\%)$ & 18.25 & 13.77 & 18.18 & 13.75 & $0.4 \%$ & $0.2 \%$ \\
\hline $\boldsymbol{V}_{\text {OC }}(\mathbf{m V})$ & 650.0 & 559.3 & 642.0 & 545.6 & $-0.2 \%$ & $0.1 \%$ \\
\hline$J_{\text {SC }}\left(\mathbf{m A} / \mathbf{c m}^{\mathbf{2}}\right)$ & 38.41 & 32.79 & 38.48 & 32.77 & $1.3 \%$ & $2.5 \%$ \\
\hline $\boldsymbol{F F}(\mathbf{\%})$ & 73.11 & 75.10 & 73.60 & 76.90 & $-0.7 \%$ & $-2.3 \%$ \\
\hline
\end{tabular}

\section{RESULTS AND DISCUSSION}

\subsection{Mapping the effects of dislocation clusters}

Fig. 4 shows contour plots of efficiency as a function of $D D_{\text {cluster }}$ and the dislocated area fraction $\left(A_{\mathrm{DL}}\right)$ of the cell, for the cases of a low and high $N_{\mathrm{DL}}$ (top and bottom panels) in both PERT and heterojunction cells (left and right panels). The low value of $N_{\text {DL }}$ is $10^{3}$ $\mathrm{cm}^{-1}$ and the high value is $10^{7} \mathrm{~cm}^{-1}$. See Supplementary Materials for contour plots of additional values of $N_{\mathrm{DL}}$ and additional device performance parameters-open-circuit voltage $\left(V_{\mathrm{OC}}\right)$, short-circuit current $\left(J_{\mathrm{SC}}\right)$, and fill factor $(F F)$. It is clear from these results that all three factors - $A_{\mathrm{DL}}, D D_{\text {cluster, }}$ and $N_{\mathrm{DL}}$ - are critical in determining the effect of dislocations on device performance.

To explicitly show the effect of clustering, we plot lines of constant average dislocation density in Fig. $\mathbf{4}$ as blue dashed lines. These contours reveal the relative effect of clustered $v s$. homogeneously distributed dislocations: at low $N_{\mathrm{DL}}$, these contours follow the contours of cell efficiency, while at high $N_{\text {DL }}$ they do not. Hence, at high $N_{\text {DL }}$, higher cell efficiencies are reached if the dislocations are clustered than if they are homogeneously distributed. For example, the circles in the lower left plot demonstrate that while a cell with $A_{\mathrm{DL}}=100 \%$ and $D D_{\text {cluster }}=10^{5} \mathrm{~cm}^{-2}$ has the same average dislocation density as a cell with $A_{\mathrm{DL}}=10 \%$ and $D D_{\text {cluster }}=10^{6} \mathrm{~cm}^{-2}$, cell efficiency is significantly different. Generally, clustering improves performance, however we reiterate that the effect is more pronounced at higher $N_{\text {DL }}$. This result contrasts with earlier simulations by Sopori, which suggested clustered dislocations were worse [31,32]. Sopori obtained local $J$ - $V$ characteristics by fitting the two-diode equation for small mesa diodes on a wafer [33] and carried out parallel-diode modeling for characteristics of one defect-free area and one area containing a dislocation cluster. Discrepancies between those results and the results presented here could be due to the two-diode fitting procedure used, effects in the dislocated area of the measured cell that were unrelated to the dislocations, the assumptions made about lateral series resistance, differences in the device architecture used, or length-scale effects (i.e., the clustering described by Sopori better describing an increase in dislocation density within a cluster rather than the spatial distribution of discrete clusters across a wafer). 

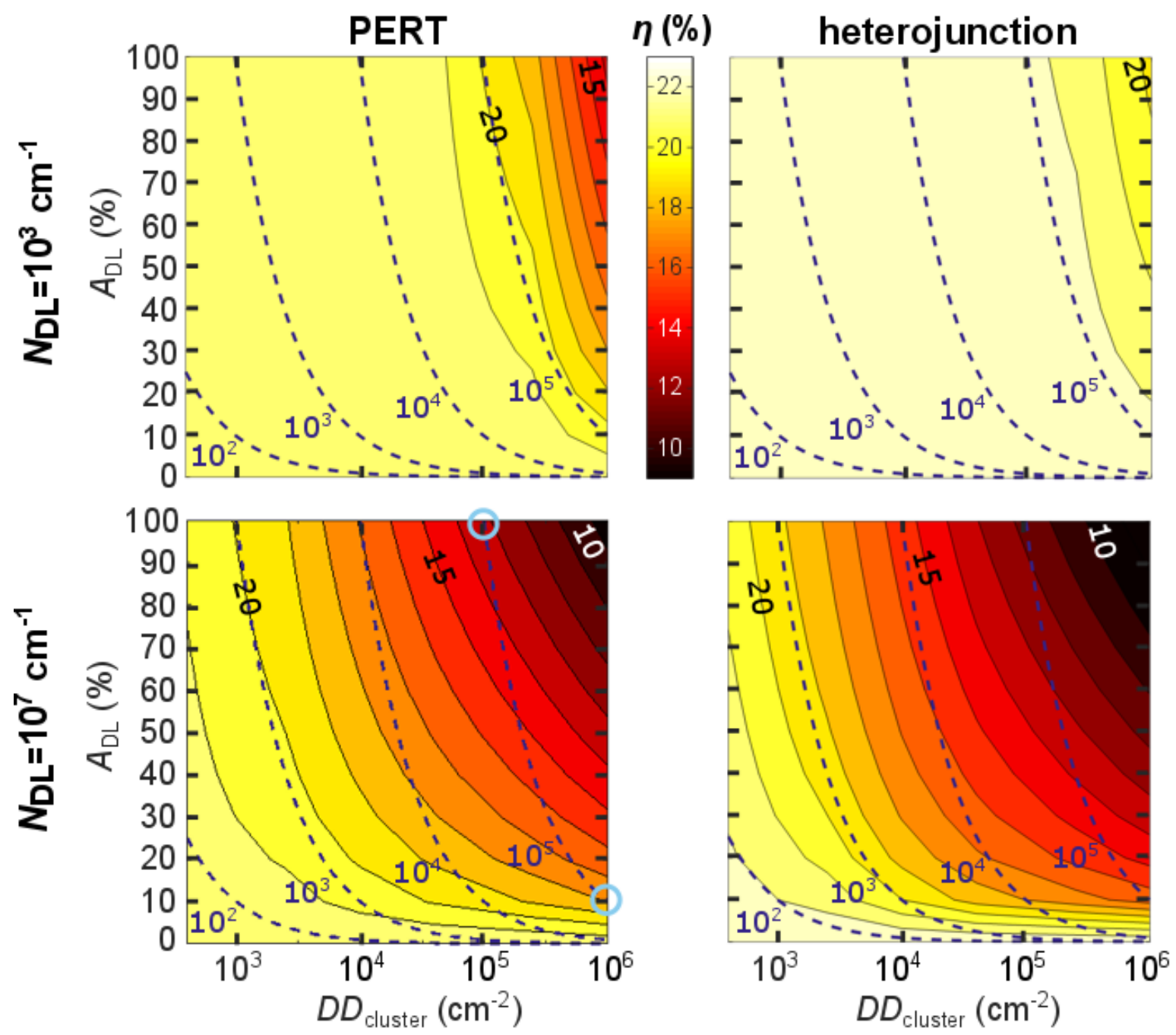

FIG. 4. Simulated cell efficiency of PERT (left column) and heterojunction (right column) cells as a function of $D D_{\text {cluster }}\left(x\right.$-axis) and $A_{\mathrm{DL}}$ ( $y$-axis) for low (top row) and high (bottom row) $N_{\mathrm{DL}}$. The heterojunction is more robust to the presence of dislocations at low $N_{\mathrm{DL}}$ and more sensitive to the presence of dislocations at high $N_{\text {DL. }}$ Blue dashed lines indicate constant average dislocation density in the cell, showing that clustered defects have less impact than homogeneously distributed ones. For additional values of $N_{\mathrm{DL}}$ and additional performance parameters $\left(V_{\mathrm{OC}}, J_{\mathrm{SC}}, \mathrm{FF}\right)$, see supplementary materials.

For low $N_{\text {DL }}$ (top row of Fig. 4), cell efficiency does not improve when $D D_{\text {cluster }}$ is reduced below about $10^{5} \mathrm{~cm}^{-2}$. Below this value, recombination outside the base dominates the total recombination losses. The exact value for this plateau will depend on the specific device design. The heterojunction cell is more robust to the presence of dislocations than the PERT cell at low $N_{\text {DL }}$ but performs worse at high $N_{\text {DL }}$. This mirrors an effect seen with our simulations of grain boundaries. We attribute the robustness at low $N_{\mathrm{DL}}$ to the fact that the silicon wafer in the optimized [21] heterojunction cell is more lightly doped than in the optimized [21] PERT cell $\left(6 \times 10^{14} \mathrm{~cm}^{-3}\right.$ vs. $\left.7 \times 10^{15} \mathrm{~cm}^{-3}\right)$, while the injection level at the maximum power point is much higher $\left(5 \times 10^{14} \mathrm{~cm}^{-3} v s .10^{13} \mathrm{~cm}^{-3}\right)$ due to reduced recombination in the emitter, at the front surface, and in the front contact. This combination leads to a smaller fraction of the mid-gap recombination centers being occupied by majority carriers and reduced charging of the dislocations. This reduced charging decreases the size of the depletion region around the dislocations, reducing recombination at the defects. In contrast, at high $N_{\text {DL }}$, recombination through the dislocation pushes the heterojunction into low injection. Then, the lighter doping leads to a 
larger depletion region around the dislocations and to higher recombination. The injection level and relative size of the depletion region in these two cases is illustrated in Fig. 5. We note that while heterojunction architectures are typically reserved for $\mathrm{Cz}-\mathrm{Si}$, these architectures can also maximize the efficiency of high-performance mc-Si and other highlifetime materials whose performance is limited by dislocations.
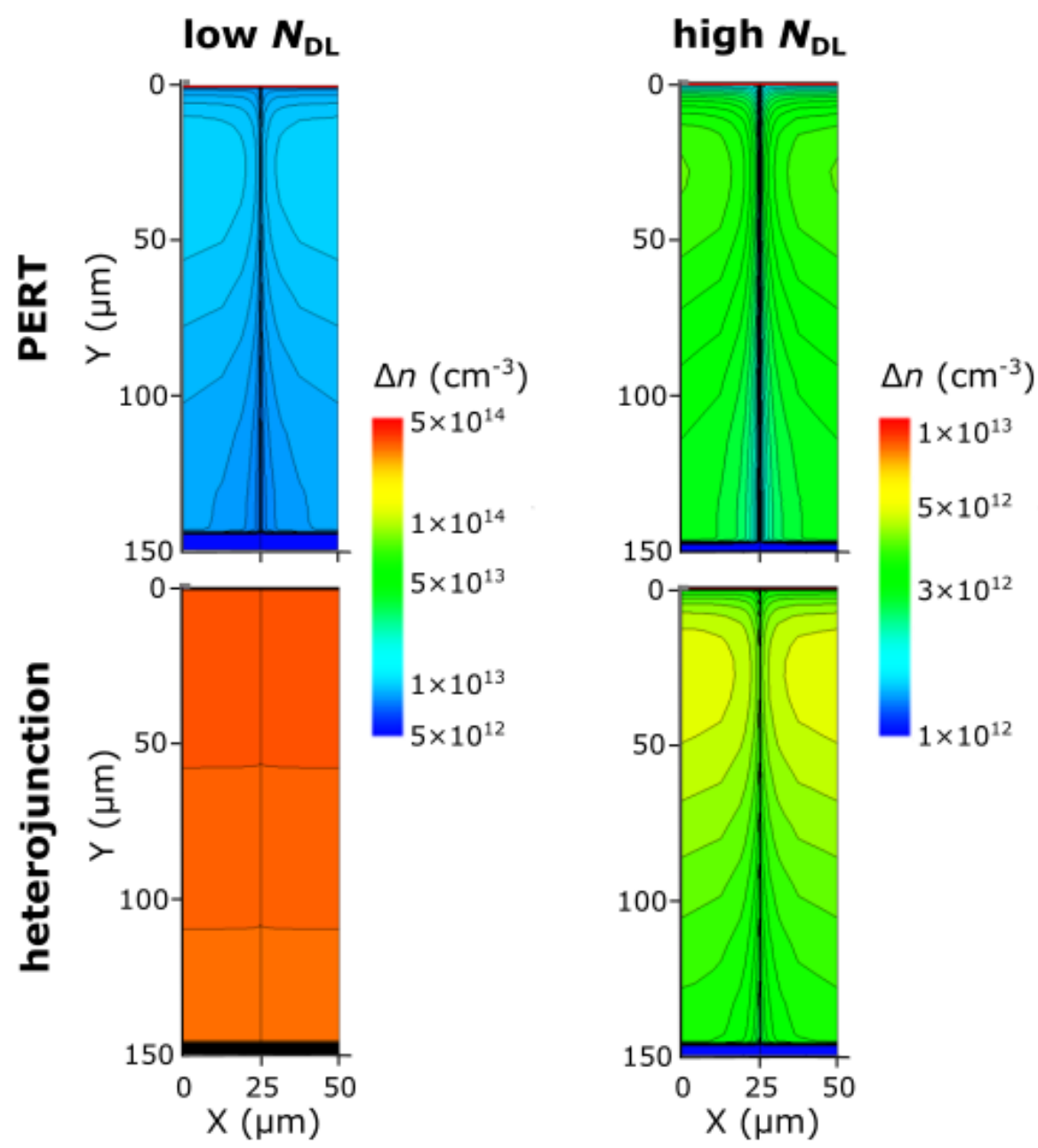

FIG. 5. The excess carrier density plotted in the neighborhood of a dislocation line, simulated at the maximum power point of the PERT (top row) and the heterojunction (bottom row) cells. The heterojunction cell is in higher injection for low $N_{\mathrm{DL}}\left(10^{3} \mathrm{~cm}^{-1}\right.$, left column), leading to less charging of the dislocation, a smaller depletion region around the dislocation, and less recombination at the dislocation. Both devices are in low-injection for high $N_{\mathrm{DL}}\left(10^{6} \mathrm{~cm}^{-1}\right.$, right column), leading to similar charging and a larger depletion region in the more lightly doped heterojunction device. The left scale bar is the low $N_{\mathrm{DL}}$ simulations and the right scale bar is for the high $N_{\text {DL }}$ simulations.

Both the PERT and the heterojunction cells can achieve efficiencies approaching those of monocrystalline silicon. For low $N_{\mathrm{DL}}$, this occurs for average dislocation densities between $10^{4}$ and $10^{5} \mathrm{~cm}^{-2}$ for the PERT cell and above $10^{5} \mathrm{~cm}^{-2}$ for the heterojunction. For high $N_{\mathrm{DL}}$, it occurs for the PERT cell only below a dislocation density of $10^{4} \mathrm{~cm}^{-2}$ with an area fraction of $10 \%$ or below $30 \%$ area fraction for a dislocation density of $10^{3} \mathrm{~cm}^{-2}$. For the heterojunction, these values are even lower with an area fraction of $10 \%$ required for a dislocation density of $10^{3} \mathrm{~cm}^{-2}$. 


\subsection{Evaluating experimental devices and materials}

Our analysis to this point has assumed that the dislocation-free regions of the cells are high-lifetime (1 ms) and contain no other defects. However, our model can also be used to predict the performance of materials, such as high-performance mc-Si, that contain other defects outside of the dislocation clusters. We demonstrate one such approach here with the world record high-performance mc-Si solar cell from Trina Solar [34], which has a passivated emitter and rear (PERC) structure. First, we take a photoluminescence (PL) image of the cell with a resolution of $155 \mu \mathrm{m} /$ pixel (Fig. 6). From this image, we can obtain both the local dislocation density, and the local recombination strength of these dislocation clusters.

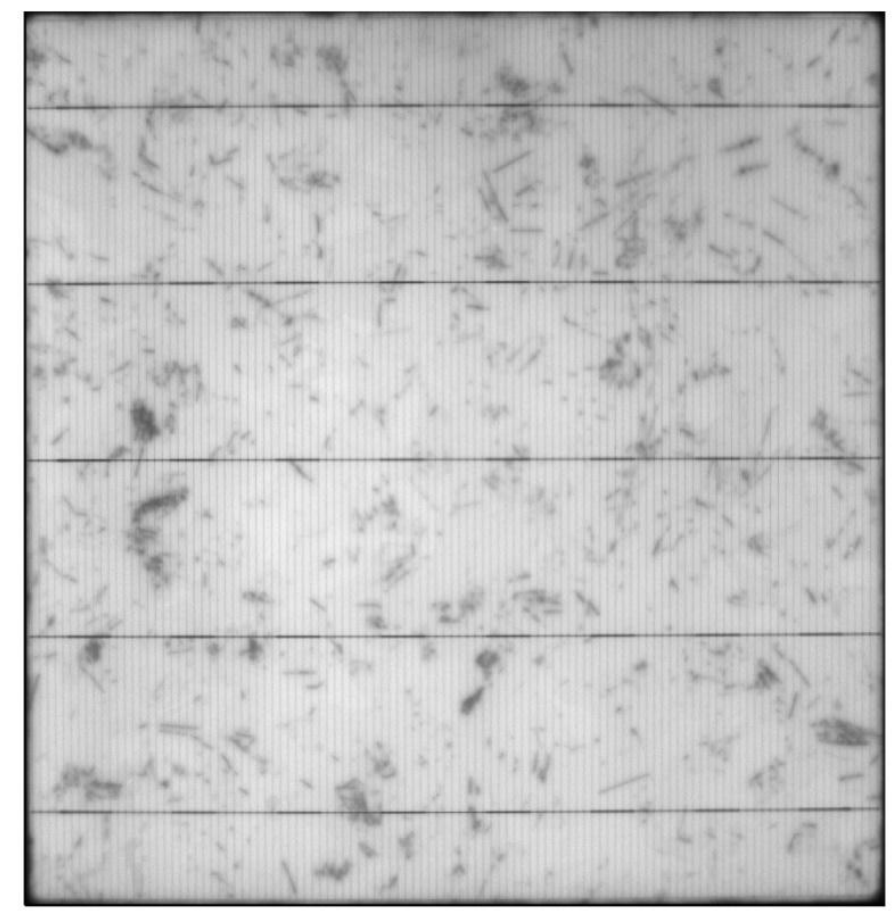

FIG. 6. Photoluminescence image of Trina Solar's high-performance mc-Si PERC record [34,35] solar cell at an internal photon flux density of $5.747 \times 10^{16} \mathrm{~cm}^{-2} \mathrm{~s}^{-1}$ (resolution: $155 \mu \mathrm{m} /$ pixel, total area: $156 \times 156 \mathrm{~mm}^{2}$ ).

To obtain dislocation density, we use a method similar to one developed for quantifying dislocation density from low-resolution image of a dislocation-etched silicon wafer [36], whereby we threshold the image so that dislocation clusters appear black and nondislocated regions appear white. To use this approach with a PL image assumes that areas with much lower lifetime than those around them contain dislocations. With the PL image, instead of calibrating with a higher resolution image as in [36], we assume that each black pixel contains a certain number of dislocations. We choose 100 dislocations per pixel in this case in order to have upper and lower detection limits that match the range of dislocation densities measured on a representative $2.7 \mathrm{~mm} \times 2.0 \mathrm{~mm}$ region of another wafer from the same ingot. We then average the number of black pixels over an area containing 100 pixels $\left(2.4 \mathrm{~mm}^{2}\right)$ to determine the average dislocation density in each of these regions.

To obtain an estimate for $N_{\mathrm{DL}}$ in each of the regions containing dislocation clusters, we average the PL contrast (local PL intensity/maximum PL intensity) over each region. By correlating this contrast to simulations of total radiative recombination in dislocation clusters compared to total radiative recombination in dislocation-free areas, we determine the $N_{\mathrm{DL}}$ associated with each dislocation cluster. These values range from $10^{3} \mathrm{~cm}^{-1}$ to $4 \times$ 
$10^{4} \mathrm{~cm}^{-1}$ for this cell. The low recombination strength of the dislocation clusters enables the high efficiency of this cell.

By selecting simulated $J-V$ curves with the same combination of dislocation density and $N_{\text {DL }}$, we now have $J-V$ curves for every dislocation cluster in the experimental cell. We can use these curves in two ways. First, we add the measured distribution of dislocation clusters to a simulation of a dislocation-free cell to show that it reproduces the experimental $J-V$ curve. Deng et al. simulated the experimental device previously using a lifetime model with shallow and deep energy levels to fit the measured injection dependence due to the presence of grain boundaries and dislocations [35]. Here, we use a similar model with identical peak doping profiles and depths for the emitter and the Al$\mathrm{BSF}$ around the local point contacts but slightly different profiles. To compensate for this discrepancy, the background lifetime is changed slightly from the values used by Deng et $a l$. Specifically, they used a deep defect at mid-gap and a shallow defect at $E_{\mathrm{C}}-E_{\mathrm{t}}=0.1 \mathrm{eV}$ with $\tau_{\text {n,deep }}=250 \mu \mathrm{s}, \tau_{\mathrm{p} \text {,deep }}=2500 \mu \mathrm{s}$, and $\tau_{\mathrm{n} \text {,shallow }}=\tau_{\mathrm{p} \text {,shallow }}=7.6 \mu \mathrm{s}$. In this work, these values are modified to $\tau_{\mathrm{n} \text {,deep }}=100 \mu \mathrm{s}, \tau_{\mathrm{p} \text {,deep }}=6000 \mu \mathrm{s}$, and and $\tau_{\mathrm{n} \text {, shallow }}=\tau_{\mathrm{p} \text {, shallow }}=3.75$ $\mu \mathrm{s}$. Excellent agreement is obtained for both simulations with the experimental results (less than $0.5 \%$ rel difference in $\eta, V_{\mathrm{OC}}, J_{\mathrm{SC}}$, and $F F$ ).

For the dislocation-free simulation, we reduce the concentration of shallow defects (increase the lifetime associated with the shallow defect level) by the area fraction of the wafer that contains dislocations, obtaining $\tau_{\mathrm{n} \text {,shallow }}{ }^{\prime}=\tau_{\mathrm{p} \text {,shallow }}{ }^{\prime}=4.29 \mu \mathrm{s}$. The minority carrier (electron) lifetime associated with the deep defect is increased as well. The new value $\left(\tau_{\text {n,deep }}\right)$ is chosen so that the total recombination in the device with $\tau_{\mathrm{n} \text {,deep }}$ ' in the nondislocated areas and $\tau_{\mathrm{n} \text {,deep, } i}$ in each dislocated region to give the measured PL contrast in that region is equal to the total recombination for the original values of $\tau_{\mathrm{n} / \mathrm{p} \text {,shallow }}$ and $\tau_{\mathrm{n} / \mathrm{p} \text {,deep }}$. This calculation gives $\tau_{\mathrm{n} \text {,deep }}{ }^{\prime}=200 \mu \mathrm{s}$, with $\tau_{\mathrm{p} \text {,deep }}{ }^{\prime}=\tau_{\mathrm{p} \text {,deep }}=6000 \mu \mathrm{s}$. When the dislocation distribution measured on the experimental cell is added to this simulation using (1), excellent agreement is obtained with the experimental $J$ - $V$ curve as shown in Fig. 7 and Table II (first and second rows), confirming the ability of the model to correctly capture the effect of dislocations on solar cell performance.

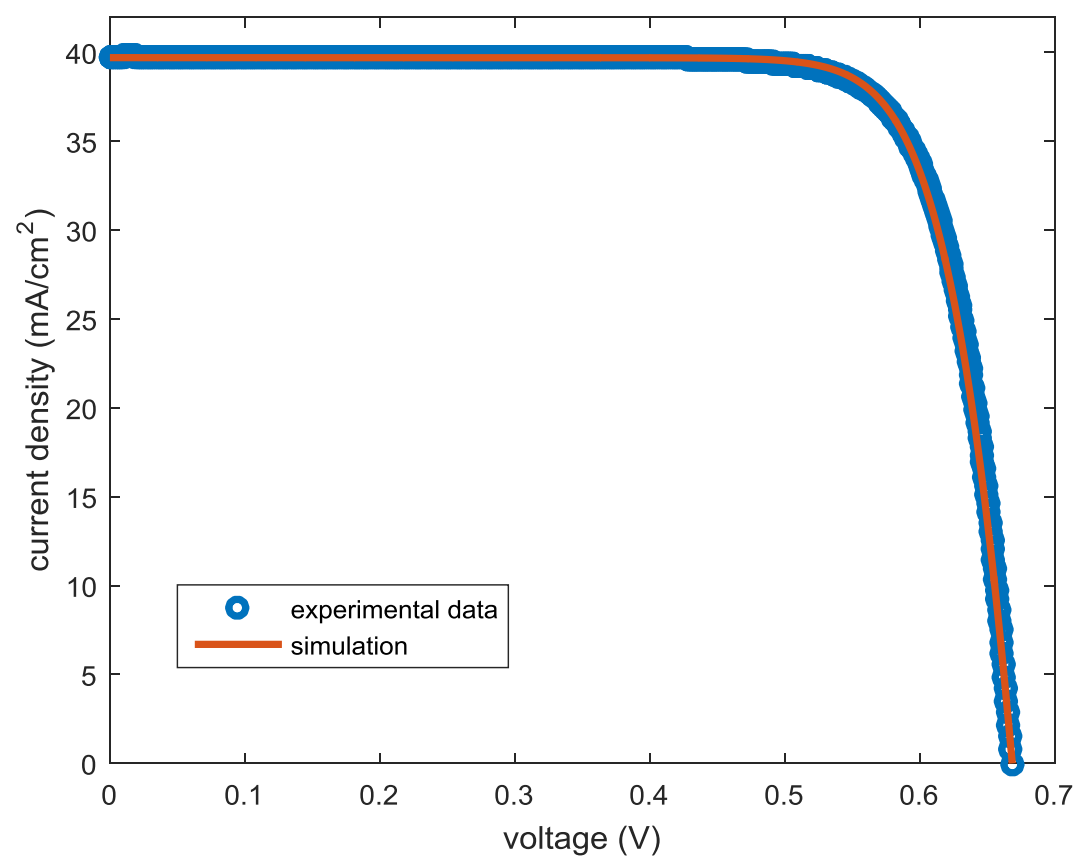

FIG. 7. Comparison of experimental (blue circles) and simulated (red line) $J$ - $V$ curves for the world record mc-Si solar cell. The simulated curve is obtained by adding the measured distribution of dislocation clusters to a simulation of a dislocation-free cell with similar background lifetime and device architecture. Excellent agreement is obtained. 
We can then subtract the effect of dislocations from the measured $J-V$ curve using the same weighted average approach described in Section 2. Specifically, we use the formula:

$$
J_{\text {clean }}(V)=\frac{J_{\text {cell }}(V)-\sum A_{\mathrm{DL}} \times J_{\mathrm{DL}}(V)}{1-\sum A_{\mathrm{DL}}}
$$

where $J_{\text {clean }}(V)$ is the calculated current as a function of voltage for a dislocation-free solar cell, $J_{\text {cell }}(V)$ is the measured current as a function of voltage, $A_{\mathrm{DL}}$ is the fractional area of each measured dislocation cluster, and $J_{\mathrm{DL}}(V)$ is the simulated current as a function of voltage for the combination of dislocation density and $N_{\mathrm{DL}}$ extracted for each measured dislocation cluster. For $J_{\mathrm{DL}}$ here and the fitting of $N_{\mathrm{DL}}$ described above, the PERC model that matches the experimental device is used rather than the PERT model described in Section 2.

The results for the world record mc-Si solar cell are shown in Table II. Due to the high quality of the wafer used for this cell, the impact of dislocations is limited. We calculate that if the cell were dislocation free, the efficiency would increase $0.26 \%$ abs, mostly due to a $0.49 \mathrm{~mA} / \mathrm{cm}^{2}$ increase in $J_{\mathrm{SC}}$. We attribute the large difference in $J_{\mathrm{SC}}$, accompanied by a small difference in $V_{\mathrm{OC}}$ to the highly asymmetric capture cross-sections for majority and minority carriers at the dislocations. This asymmetry means that recombination is dominated by the dislocation at $J_{\mathrm{SC}}$ conditions and by other parts of the device at $V_{\mathrm{OC}}$.

TABLE II: Measured and dislocation-free performance of world record mc-Si solar cell.

\begin{tabular}{|c|c|c|c|c|}
\hline & $\boldsymbol{\eta}(\boldsymbol{\%})$ & $V_{\text {OC }}(\mathbf{m V})$ & $J_{\text {SC }}\left(\mathbf{m A} / \mathbf{c m}^{2}\right)$ & $\boldsymbol{F F}(\boldsymbol{\%})$ \\
\hline $\begin{array}{c}\text { Measured } \\
\text { (experimental) }\end{array}$ & 21.25 & 667.8 & 39.78 & 79.97 \\
\hline $\begin{array}{c}\text { Simulated } \\
\text { (dislocations added) }\end{array}$ & 21.33 & 668.5 & 39.71 & 80.35 \\
\hline $\begin{array}{c}\text { Simulated } \\
\text { (lifetime model) }\end{array}$ & 21.27 & 667.3 & 39.97 & 79.73 \\
\hline $\begin{array}{c}\text { Dislocation-free } \\
\text { (simulated, lifetime model) }\end{array}$ & 21.53 & 668.9 & 40.10 & 80.27 \\
\hline $\begin{array}{c}\text { Dislocation-free } \\
\text { (simulated, dislocations removed) }\end{array}$ & 21.51 & 668.3 & 40.20 & 80.06 \\
\hline $\begin{array}{c}\text { High-lifetime, dislocations added } \\
\text { (simulated) }\end{array}$ & 21.73 & 668.1 & 40.04 & 81.22 \\
\hline $\begin{array}{c}\text { High-lifetime, dislocation-free } \\
\text { (simulated) }\end{array}$ & 21.92 & 668.5 & 40.35 & 81.26 \\
\hline \multicolumn{2}{|c|}{} & & & \\
\hline
\end{tabular}

Once the dislocation-free $J-V$ curve is obtained, the device performance can be calculated for any arbitrary distribution of dislocation clusters with any distribution of $N_{\mathrm{DL}}$. These calculations would show not only the limits of performance but the effect of smaller reductions in dislocation density and decoration as well as changes in dislocation distribution.

We also simulate the effect of increased lifetime in the dislocation-free region $\left(\tau_{\mathrm{n}, \mathrm{deep}}{ }^{\prime}=\right.$ $1000 \mu \mathrm{s})$ with the same distribution of dislocations. The simulated efficiency in this case is $21.73 \%$, mostly due to increased $F F$. With $\tau_{\text {n,deep }}$ "' $=1000 \mu$ s and no dislocations, efficiency further increases to $21.92 \%$ primarily with high $F F$ and $J_{\mathrm{SC}}$. The remaining 
difference in efficiency between this simulated device and experimental results for CZ-Si in a similar architecture [37] are due to the low value of $\tau_{\mathrm{n} / \mathrm{p} \text {,shallow }}(i . e$. the presence of grain boundaries). Thus, the performance of this cell is limited by dislocations, grain boundaries, and bulk point defects. We note, however, that our simulations indicate that similar material in a higher efficiency architecture, like the theoretical GaP/Si heterojunction simulated here, would likely perform better than CZ-Si in even the best commercial PERC architecture.

\section{CONCLUSION}

We show that the device performance impacts of dislocations depends critically on three parameters: the area fraction of the solar cell that contains dislocation clusters, the dislocation density within these clusters, and the line density of mid-gap recombination centers along each dislocation. We also demonstrate how using a weighted average of the external current as a function of external voltage for dislocation clusters and non-dislocated regions is appropriate for macroscopic dislocation clusters and can be used to determine the effect of dislocations (and defect reduction or mitigation) on experimental devices.

With three-dimensional numerical TCAD simulations, we map performance as a function of the three relevant parameters to predict the materials properties necessary for high-efficiency PERT and heterojunction solar cells. We find that for relatively clean (undecorated) or well-passivated dislocations, there is a wide range of dislocation densities and dislocated area fractions that enable high efficiency. As an example, we investigate the impact of dislocations on the recent record cell made with high-performance mc-Si. We use these results to validate our dislocation model for solar cell devices and explore the aspects of material quality (dislocation clusters, grain boundaries, and bulk point defects) that limit device performance. Finally, we show that higher-injection architectures are more tolerant to the presence of dislocations because they reduce dislocation charging and thereby the size of the depletion region around the dislocations. While we note that lighter doping, which can contribute to a higher relative injection level, is detrimental at high defect concentrations, the improvement at low defect concentrations has more practical significance because these are the conditions necessary for high-efficiency. The performance of mc-Si similar to that used for the world record mc-Si cell in such an architecture with a higher efficiency potential than PERC (e.g., heterojunction device architecture) would likely exceed the efficiency of CZ-Si PERC.

\section{ACKNOWLEDGEMENTS}

This material is based upon work primarily supported by the Engineering Research Center Program of the National Science Foundation and the Office of Energy Efficiency and Renewable Energy of the Department of Energy (DOE) under NSF Cooperative Agreement No. EEC-1041895. This work was supported in part by the DOE under Award Numbers DE-EE0005314 and DE-EE0006335. D. Berney Needleman acknowledges the support of the Department of Defense (DoD) through the National Defense Science \& Engineering Graduate Fellowship (NDSEG) program.

\section{REFERENCES}

[1] S. Pizzini, Chemistry and physics of segregation of impurities at extended defects in silicon, Phys. Status Solidia. 171 (1999) 123-132. 
[2] A. Bentzen, A. Holt, R. Kopecek, G. Stokkan, J.S. Christensen, B.G. Svensson, Gettering of transition metal impurities during phosphorus emitter diffusion in multicrystalline silicon solar cell processing, J. Appl. Phys. 99 (2006) 93509. doi:10.1063/1.2194387.

[3] O. Schultz, S.W. Glunz, S. Riepe, G.P. Willeke, High-efficiency solar cells on phosphorus gettered multicrystalline silicon substrates, Prog. Photovolt. Res. Appl. 14 (2006) 711-719. doi:10.1002/pip.736.

[4] L.J. Geerligs, Y. Komatsu, I. Röver, K. Wambach, I. Yamaga, T. Saitoh, Precipitates and hydrogen passivation at crystal defects in n- and p-type multicrystalline silicon, J. Appl. Phys. 102 (2007) 93702. doi:10.1063/1.2800271.

[5] T. Trupke, J. Nyhus, J. Haunschild, Luminescence imaging for inline characterisation in silicon photovoltaics, Phys. Status Solidi RRL - Rapid Res. Lett. 5 (2011) 131-137. doi:10.1002/pssr.201084028.

[6] M. Rinio, A. Yodyungyong, S. Keipert-Colberg, D. Borchert, A. MontesdeocaSantana, Recombination in ingot cast silicon solar cells, Phys. Status Solidi A. 208 (2011) 760-768. doi:10.1002/pssa.201084022.

[7] Y.M. Yang, A. Yu, B. Hsu, W.C. Hsu, A. Yang, C.W. Lan, Development of highperformance multicrystalline silicon for photovoltaic industry, Prog. Photovolt. Res. Appl. 23 (2015) 340-351. doi:10.1002/pip.2437.

[8] F. Schindler, B. Michl, A. Kleiber, H. Steinkemper, J. Schon, W. Kwapil, P. Krenckel, S. Riepe, W. Warta, M.C. Schubert, Potential Gain in Multicrystalline Silicon Solar Cell Efficiency by n-Type Doping, IEEE J. Photovolt. 5 (2015) 499-506. doi:10.1109/JPHOTOV.2014.2377554.

[9] J. Schön, F. Schindler, W. Kwapil, M. Knörlein, P. Krenckel, S. Riepe, W. Warta, M.C. Schubert, Identification of the most relevant metal impurities in mc n-type silicon for solar cells, Sol. Energy Mater. Sol. Cells. 142 (2015) 107-115. doi:10.1016/j.solmat.2015.06.028.

[10] L. Gong, F. Wang, Q. Cai, D. You, B. Dai, Characterization of defects in mono-like silicon wafers and their effects on solar cell efficiency, Sol. Energy Mater. Sol. Cells. 120, Part A (2014) 289-294. doi:10.1016/j.solmat.2013.09.020.

[11] C.C. Hsieh, Y.C. Wu, A. Lan, H.P. Hsu, C. Hsu, C.W. Lan, Comparison of defect formations in solar silicon growth from small random and large oriented seeds, J. Cryst. Growth. 419 (2015) 1-6. doi:10.1016/j.jcrysgro.2015.02.087.

[12] E. Kobayashi, Y. Watabe, R. Hao, T.S. Ravi, High efficiency heterojunction solar cells on n-type kerfless mono crystalline silicon wafers by epitaxial growth, Appl. Phys. Lett. 106 (2015) 223504. doi:10.1063/1.4922196.

[13] H. Wagner, M. Müller, G. Fischer, P.P. Altermatt, A simple criterion for predicting multicrystalline Si solar cell performance from lifetime images of wafers prior to cell production, J. Appl. Phys. 114 (2013) 54501. doi:10.1063/1.4817272.

[14] M. Muller, P.P. Altermatt, H. Wagner, G. Fischer, Sensitivity Analysis of Industrial Multicrystalline PERC Silicon Solar Cells by Means of 3-D Device Simulation and Metamodeling, IEEE J. Photovolt. 4 (2014) 107-113. doi:10.1109/JPHOTOV.2013.2287753.

[15] D. Berney Needleman, H. Wagner, P.P. Altermatt, T. Buonassisi, Assessing the Device-performance Impacts of Structural Defects with TCAD Modeling, Energy Procedia. 77 (2015) 8-14. doi:10.1016/j.egypro.2015.07.003.

[16] M. Kittler, W. Seifert, Two types of electron-beam-induced current behaviour of misfit dislocations in $\mathrm{Si}(\mathrm{Ge})$ : experimental observations and modelling, Mater. Sci. Eng. B. 24 (1994) 78-81. 
[17] V. Kveder, M. Kittler, W. Schröter, Recombination activity of contaminated dislocations in silicon: A model describing electron-beam-induced current contrast behavior, Phys. Rev. B. 63 (2001). doi:10.1103/PhysRevB.63.115208.

[18] G. Stokkan, S. Riepe, O. Lohne, W. Warta, Spatially resolved modeling of the combined effect of dislocations and grain boundaries on minority carrier lifetime in multicrystalline silicon, J. Appl. Phys. 101 (2007) 53515. doi:10.1063/1.2435815.

[19] Sentaurus Device User Guide, Synopsys, 2013.

[20] D. Berney Needleman, H. Wagner, P.P. Altermatt, T. Buonassisi, Three-Dimensional TCAD Modeling of Grain Boundaries in High-Efficiency Silicon Solar Cells, IEEE J. Photovolt. (2016). doi:10.1109/JPHOTOV.2016.2549739.

[21] H. Wagner, T. Ohrdes, A. Dastgheib-Shirazi, B. Puthen-Veettil, D. König, P.P. Altermatt, A numerical simulation study of gallium-phosphide/silicon heterojunction passivated emitter and rear solar cells, J. Appl. Phys. 115 (2014) 44508. doi:10.1063/1.4863464.

[22] A. Augusto, D. Pera, H.J. Choi, P. Bellanger, M.C. Brito, J. Maia Alves, A.M. Vallera, T. Buonassisi, J.M. Serra, Residual stress and dislocations density in silicon ribbons grown via optical zone melting, J. Appl. Phys. 113 (2013) 83510. doi:10.1063/1.4793319.

[23] D.M. Powell, J. Hofstetter, D.P. Fenning, R. Hao, T.S. Ravi, T. Buonassisi, Effective lifetimes exceeding $300 \mu$ s in gettered p-type epitaxial kerfless silicon for photovoltaics, Appl. Phys. Lett. 103 (2013) 263902. doi:10.1063/1.4844915.

[24] R. Hao, T. s. Ravi, V. Siva, J. Vatus, D. Miller, J. Custodio, K. Moyers, High quality kerfless silicon mono-crystalline wafers and cells by high throughput epitaxial growth, in: Symp. - Film-Silicon Sci. Technol., 2014: p. mrss14-1666-a05-01 (11 pages). doi:10.1557/opl.2014.716.

[25] N. Stoddard, B. Gründig-Wendrock, A. Krause, D. Oriwol, M. Bertoni, T.U. Naerland, I. Witting, L. Sylla, On the potential and limits of large area seeding for photovoltaic silicon, J. Cryst. Growth. (n.d.). doi:10.1016/j.jcrysgro.2016.04.056.

[26] M. Kivambe, D.M. Powell, S. Castellanos, M. Ann Jensen, A.E. Morishige, K. Nakajima, K. Morishita, R. Murai, T. Buonassisi, Minority-carrier lifetime and defect content of n-type silicon grown by the noncontact crucible method, J. Cryst. Growth. 407 (2014) 31-36. doi:10.1016/j.jcrysgro.2014.08.021.

[27] K. Nakajima, R. Murai, S. Ono, K. Morishita, M.M. Kivambe, D.M. Powell, T. Buonassisi, Shape and quality of Si single bulk crystals grown inside Si melts using the noncontact crucible method, Jpn. J. Appl. Phys. 54 (2015) 15504. doi:10.7567/JJAP.54.015504.

[28] Z. Xiong, Z. Zhang, H. Ye, S. Fu, P.P. Altermatt, Z. Feng, P.J. Verlinden, High performance multicrystalline wafers with lifetime of $400 \mu$ s at industrial scale, in: Photovolt. Spec. Conf. PVSC 2015 IEEE 42nd, 2015: pp. 1-4. doi:10.1109/PVSC.2015.7355615.

[29] J. Wong, Griddler: Intelligent computer aided design of complex solar cell metallization patterns, in: Photovolt. Spec. Conf. PVSC 2013 IEEE 39th, 2013: pp. 0933-0938. doi:10.1109/PVSC.2013.6744296.

[30] H. Haug, M.S. Wiig, R. Søndenå, J. Wong, Simulating the effect of lifetime nonuniformity on solar cell performance using cmd-PC1D 6 and Griddler 2, Energy Procedia. (under review).

[31] B.L. Sopori, Defect Clusters in Silicon: Impact on the Performance of Large-Area Devices, Mater. Sci. Forum. 258-263 (1997) 527-534. doi:10.4028/www.scientific.net/MSF.258-263.527. 
[32] B. Sopori, V. Budhraja, P. Rupnowski, S. Johnston, N. Call, H. Moutinho, M. AlJassim, Defect clusters in multicrystalline silicon: Their nature and influence on the solar cell performance, in: Photovolt. Spec. Conf. PVSC 2009 34th IEEE, 2009: pp. 1969-1974. http://ieeexplore.ieee.org/xpls/abs_all.jsp?arnumber=5411527 (accessed August 16, 2013).

[33] B.L. Sopori, Fabrication of diode arrays for photovoltaic characterization of silicon substrates, Appl. Phys. Lett. 52 (1988) 1718-1720. doi:10.1063/1.99027.

[34] M.A. Green, K. Emery, Y. Hishikawa, W. Warta, E.D. Dunlop, Solar cell efficiency tables (version 47), Prog. Photovolt. Res. Appl. 24 (2016) 3-11. doi:10.1002/pip.2728.

[35] W. Deng, F. Ye, Z. Xiong, D. Chen, W. Guo, Y. Chen, Y. Yang, P.P. Altermatt, Z. Feng, P.J. Verlinden, Development of high-efficiency industrial p-type multicrystalline PERC solar cells with efficiency greater than $21 \%$, Energy Procedia. (under review).

[36] D. Berney Needleman, H. Choi, D.M. Powell, T. Buonassisi, Rapid dislocationdensity mapping of as-cut crystalline silicon wafers, Phys. Status Solidi RRL - Rapid Res. Lett. 7 (2013) 1041-1044. doi:10.1002/pssr.201308150.

[37] C. Battaglia, A. Cuevas, S.D. Wolf, High-efficiency crystalline silicon solar cells: status and perspectives, Energy Environ. Sci. 9 (2016) 1552-1576. doi:10.1039/C5EE03380B. 